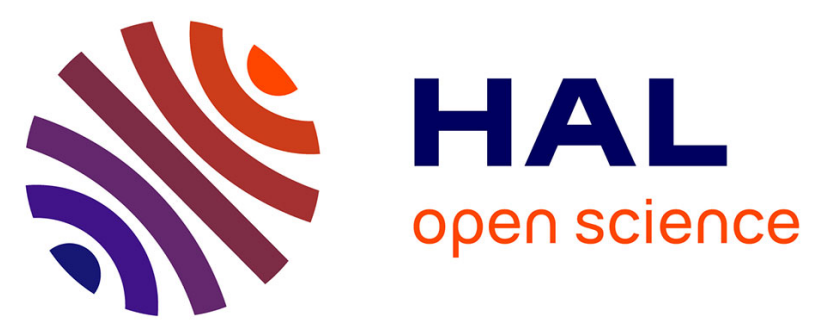

\title{
Automatic underwater fish species classification with limited data using few-shot learning
}

Sebastien Villon, Corina Iovan, Morgan Mangeas, Thomas Claverie, David Mouillot, Sébastien Villéger, Laurent Vigliola

\section{To cite this version:}

Sebastien Villon, Corina Iovan, Morgan Mangeas, Thomas Claverie, David Mouillot, et al.. Automatic underwater fish species classification with limited data using few-shot learning. Ecological Informatics, 2021, 63, pp.101320. 10.1016/j.ecoinf.2021.101320 . hal-03415715

\section{HAL Id: hal-03415715 \\ https://hal.umontpellier.fr/hal-03415715}

Submitted on 22 Nov 2021

HAL is a multi-disciplinary open access archive for the deposit and dissemination of scientific research documents, whether they are published or not. The documents may come from teaching and research institutions in France or abroad, or from public or private research centers.
L'archive ouverte pluridisciplinaire HAL, est destinée au dépôt et à la diffusion de documents scientifiques de niveau recherche, publiés ou non, émanant des établissements d'enseignement et de recherche français ou étrangers, des laboratoires publics ou privés. 
7

8

\section{Automatic underwater fish species}

classification with limited data using

few-shot learning

Sébastien Villon ${ }^{a}{ }^{*}$, Corina Iovana, Morgan Mangeasa ${ }^{a}$, Thomas Claverie ${ }^{b, c}$, David

Mouillot $^{c, d}$, Sébastien Villéger ${ }^{c}$, Laurent Vigliola ${ }^{a}$

a ENTROPIE, IRD, University of New-Caledonia, University of La Reunion, CNRS, Ifremer, Labex Corail, Noumea, New-Caledonia, France

b CUFR Mayotte, France

c MARBEC, University of Montpellier, CNRS, IRD, Ifremer, Montpellier, France

d Institut Universitaire de France, Paris, France

* Corresponding author: sebastien.villon@ird.fr 
Abstract:

Underwater cameras are widely used to monitor marine biodiversity, and the trend is increasing due to the availability of cheap action cameras. The main bottleneck of video methods now resides in the manual processing of images, a time-consuming task requiring trained experts. Recently, several solutions based on Deep Learning $(D L)$ have been proposed to automatically process underwater videos. The main limitation of such algorithms is that they require thousands of annotated images in order to learn to discriminate classes (here species). This limitation implies two issues: 1) the annotation of hundreds of common species requires a lot of efforts 2) many species are too rare to gather enough data to train a classic DL algorithm. Here, we propose to explore how

Keywords: few-shot learning, Deep learning, video, marine biodiversity 


\section{Introduction}

The world's ecosystems have entered an era of anthropogenic defaunation where human activities have triggered global decline in animal abundance, species range contraction and a new wave of species extinction [1]. This global change is threatening ecosystem services worldwide hence the stability of our food systems, economies, and health. Defaunation is more advanced in terrestrial and freshwater ecosystems than in the marine environment where it started centuries later. However, the pace of defaunation is accelerating in oceans mostly due to the advent of industrial fishing since a century ago [2]. Given this context of global changes rapidly affecting fish communities, it is imperative to monitor fish biodiversity over time, on a large scale and using nondestructive methods.

Fish biodiversity surveys in the marine environment are typically performed by divers. Although dive visual censuses provide a great deal of information on some shallow habitats, there are many limitations. First, divers are limited by depth and can hardly perform long dives to count fish below $30 \mathrm{~m}$, ignoring mesophotic habitats and deeper ecosystems. Second, divers are limited by time and generally focus their 2-4 dives per day in the most speciose hard-substrate habitats, and ignore less rich and often immense adjacent soft-bottom habitats. Third, dive surveys provide data at a slow rate so that the compilation of global fish biodiversity database takes decades of efforts by multiple teams of highly skilled taxonomic divers (e.g. [3], [4]). This is a major restriction to the necessary temporal monitoring of global marine ecosystems, although a few time series exist in some countries ${ }^{1}[5]$.

\footnotetext{
${ }_{1}^{1}$ AIMS, Long-Term Monitoring Program: Visual Census Fish Data (Great Barrier Reef) https://apps.aims.gov.au/metadata/view/5be0b340-4ade-11dc-8f56-00008a07204e
} 
Underwater videos (UV) are increasingly used [6] to overcome the limitations of diver-based surveys to quickly collect large amounts of data. For instance, more than 15,000 video stations were deployed in 58 countries in just three years for the first global assessment of the conservation status of reef sharks [7]. Furthermore, underwater video surveys can be performed in many habitats, with some example in shallow reefs [8], sandy lagoons [9], deep sea [10], and even in the pelagic ecosystem [11]. Deploying underwater video stations does not require expert taxonomists and is now quite inexpensive with the improvement of cheap action cameras since a few years. The bottleneck to analyse this data now resides in the manual processing of the videos. Indeed, manually extracting fish biodiversity and abundance data from raw videos requires unsustainable workload by highly trained taxonomic experts. Although this annotation work can be improved through citizen science [12]-[14], such time-consuming and expensive task cannot match the increasing size of datasets, up to 20,000 hours of videos for global surveys [7] and the necessary monitoring of global oceans over time.

As the demand for automatic methods to analyze underwater videos is rising, the latest generation of deep learning algorithms (DL), and in particular convolutional neural networks (CNNs) are increasingly used for species identification [14]-[17] and fish detection [18]-[21]. However, these algorithms require a large dataset of annotated images (thumbnails hereafter) in order to train a robust model, able to provide satisfying results. Therefore, this method still requires collecting an important image dataset manually annotated by experts. This is especially problematic in highly diverse faunas such as coral reef fish that encompass nearly 6500 species worldwide [22]. Furthermore, a universal pattern in species distribution, including fish communities, is that both rare and common species are found in every community, with the fraction of rare species 
more important in rich ecosystems, such as coral reefs [23], [24]. It is therefore almost impossible to gather enough thumbnails of rare species to efficiently train a deep neural network in a "classic" way, which requires thousands of images per species [25]-[27]

There are two ways to tackle this problem of lack of data. The first one consists of directly addressing the data itself, through data augmentation [28]-[30]. The second option is to change the classification algorithm. Few-shots learning (FSL) algorithms [31], [32] are designed to compute a classification task (query, noted $Q$ ) with only a few thumbnails to train (Support Sets, noted SS), and it has been increasingly studied since 2017 [33]. Few-shots learning methods are divided into three main approaches. Metric-based methods are embedding both queries $(Q)$ and support sets $(S s)$, before assigning to the query a class, according to distances computed between $Q$ and $S s$ ([34]-[36]). The second approach consists of 1) training a model on a large database, and 2) adapt this model to a new task with few examples, while not forgetting the concepts learned previously [37], [38]. Finally, optimization-based methods are designed to adapt quickly to new tasks, hence able to learn a classification task with few examples [33], [39], [40]. Optimization-based algorithms showed promising results in deep learning few-shot classification [33], [41], [42]. Such methods propose to pre-train (or "meta-train") a model with existing databases (e.g. MiniImageNet [43] , Ominglot [44]) on different tasks so it can adapt easily to a new one. For object identification, a task is defined by the classes the model has to discriminate. Once this model, called "meta-model" has been trained, it can then be tuned to operate on a new task with a very limited dataset, usually only 1-5 thumbnails per class. 
133 In this study we propose to compare the efficiency of optimization-based

134 few-shot learning and standard large dataset deep-learning methods to

135 identify coral reef fish species on images. More specifically, we aim to

136 determine how well a classic deep learning architecture trained with

137 thousands of images and the benefit of data augmentation (hereafter DL)

138 and $\mathrm{FSL}$ algorithms perform in situations where training thumbnail

139 dataset is large or limited. To achieve this, we first trained a classic DL

140 architecture built for image classification [45] on a large dataset of

14169,169 thumbnails, and on a more limited dataset of 6,320 thumbnails for

14220 coral reef fish species. Then, we trained a few-shots, optimization-

143 based learning algorithm [39] on the exact same training datasets while

144 varying the number of shots from 1 to 30 . Finally, we compared the

145 capacity of DL and FSL models to correctly identify species on an

146 independent thumbnail dataset, and modelled the asymptotic relationship

147 between classification accuracy and the number of thumbnails in the

148 training datasets for both classic DL and FSL algorithms 
Material and methods

152

Thumbnail datasets

We used three fish thumbnail datasets ( $T O, T 1$, and $T 2$ ) extracted from 175 underwater videos recorded on reefs around Mayotte Island (Western Indian Ocean) using GoPro Hero 3+ and GoPro hero 4+ cameras with a

resolution of $1920 \times 1080$ pixels. A thumbnail is defined as an image containing a single labelled fish belonging to one of the 20 most common fish species in the videos, and representing a broad range of sizes, colors, body orientations, and background (Supp. Fig. 1, Supp. Fig. 2).

TO is composed of 69,169 thumbnails extracted from 130 videos, with a range of 1,134 to 7,345 thumbnails per species (Table 1 ). T1 is composed of 6,320 thumbnails extracted from 20 videos with 40-1,436 images per species whereas T2 is composed of 13,232 thumbnails extracted from 25 videos with 55-3,896 images per species. Thumbnails size originally ranged from $55 \times 55$ pixels to $500 \times 450$ pixels, but were resized to $84 \times 84$ pixels before being processed through FS and DL algorithms.

The datasets $T 1$ and $T 2$ correspond to two real scenarii where videos were recorded during two trips in the field of a week each.

The three thumbnails datasets are fully independent, as they were extracted from videos recorded at different sites, with different conditions (weather, lighting, depth, time of the day, seascape) and on different days.

To train our DL architecture, we applied data augmentation to TO and T1. For each natural thumbnails in $T 0$ and $T 1$, we created 9 thumbnails through contrast augmentation or diminution, and horizontal flip. We then obtained augmented datasets composed of 691,690 (AT0) and 63,200 
179 (AT1) images respectively Supp. Table 1. Further details on thumbnail 180 datasets and data augmentation are given in [46].

181

182 Table 1: Number of natural thumbnails extracted from the videos to build 183 our three datasets

\begin{tabular}{|c|c|c|c|c|}
\hline Family & Species & $\begin{array}{c}\text { Training } \\
\text { dataset TO }\end{array}$ & $\begin{array}{c}\text { Training } \\
\text { dataset } \\
\text { T1 }\end{array}$ & $\begin{array}{c}\text { Test dataset } \\
\text { T2 }\end{array}$ \\
\hline Acanthuridae & $\begin{array}{l}\text { Acanthurus } \\
\text { leucosternon }\end{array}$ & 3,259 & 235 & 491 \\
\hline Acanthuridae & $\begin{array}{c}\text { Acanthurus } \\
\text { lineatus }\end{array}$ & 1,008 & 114 & 864 \\
\hline Acanthuridae & Naso brevirostris & 1,134 & 539 & 1932 \\
\hline Acanthuridae & Naso elegans & 7,345 & 1,435 & 3,896 \\
\hline Acanthuridae & $\begin{array}{c}\text { Zebrasoma } \\
\text { scopas }\end{array}$ & 4,970 & 48 & 579 \\
\hline Chaetodontidae & Chaetodon auriga & 2,134 & 737 & 502 \\
\hline Chaetodontidae & $\begin{array}{c}\text { Chaetodon } \\
\text { guttatissimus }\end{array}$ & 1,182 & 221 & 68 \\
\hline Chaetodontidae & $\begin{array}{l}\text { Chaetodon } \\
\text { trifascialis }\end{array}$ & 5,234 & 41 & 630 \\
\hline Chaetodontidae & $\begin{array}{l}\text { Chaetodon } \\
\text { trifasciatus }\end{array}$ & 4,421 & 71 & 82 \\
\hline Labridae & $\begin{array}{c}\text { Gomphosus } \\
\text { caeruleus }\end{array}$ & 3,131 & 57 & 173 \\
\hline Labridae & Halichoeres & 3,192 & 40 & 287 \\
\hline
\end{tabular}


hortulanus

\begin{tabular}{|c|c|c|c|c|}
\hline Labridae & $\begin{array}{l}\text { Thalassoma } \\
\text { hardwicke }\end{array}$ & 4,951 & 181 & 275 \\
\hline Lethrinidae & $\begin{array}{l}\text { Monotaxis } \\
\text { grandoculis }\end{array}$ & 3,893 & 797 & 1,422 \\
\hline Monacanthidae & $\begin{array}{c}\text { Oxymonacanthus } \\
\text { longirostris }\end{array}$ & 2,553 & 54 & 55 \\
\hline Pomacentridae & $\begin{array}{l}\text { Abudefduf } \\
\text { vaigiensis }\end{array}$ & 5,124 & 376 & 216 \\
\hline Pomacentridae & $\begin{array}{c}\text { Amblyglyphidodon } \\
\text { indicus }\end{array}$ & 1,188 & 636 & 1,310 \\
\hline Pomacentridae & $\begin{array}{c}\text { Chromis } \\
\text { opercularis }\end{array}$ & 1,525 & 81 & 93 \\
\hline Pomacentridae & $\begin{array}{l}\text { Chromis } \\
\text { ternatensis }\end{array}$ & 3,640 & 300 & 156 \\
\hline Pomacentridae & $\begin{array}{c}\text { Pomacentrus } \\
\text { sulfureus }\end{array}$ & 5,409 & 270 & 142 \\
\hline Zanclidae & Zanclus cornutus & 3,876 & 86 & 59 \\
\hline TOTAL & & 69,169 & 6,320 & 13,232 \\
\hline
\end{tabular}

184

188 Experimental design

189 To compare classic deep-learning and few-shot algorithms in situations of 190 large or small thumbnail datasets, we led five experiments using datasets 191 T0, T1, T2, AT0 and AT1 described in Supp. Table 1 : 
1) We trained a classic $D L$ algorithms architecture with our biggest dataset $A T O$ as a baseline for the DL accuracy;

2) We trained the same DL architecture with the same hyperparameters (e.g. model architecture and training process) but on a much more limited dataset (AT1). Hyper-parameters are the parameters defining the architecture (number of layers, number and size of convolutions, connections between layers) and the training process of a Deep Model (learning rate, neurone activation, back-propagation compotation).;

3) We trained the same DL architecture with limited datasets obtained by subsampling T0 to 250 and 500 images per class (here after "species" when we are referring to our experiments), corresponding to 2500 and 5000 thumbnails in ATO;

4) We pre-trained a FSL architecture on the 64 training classes of MiniImageNet (Supp. Fig. 3) and used T0 to build support sets (SS) with 1, 5, 15 and 30 thumbnails for each fish species;

5) We pre-trained the same FSL architecture on MiniImageNet and used the more limited T1 dataset to build support sets with 1, 5, 15 and 30 images per species.

We used ResNet 100 [45] as our classic deep-learning algorithm. Resnet is a convolutional neural network (CNN), a DL architecture which is able to both extract features from images and classify these images thanks to those features [47]. In order for a CNN to build an image classification model, the architecture is fed a large dataset, composed of pairs of labels and images. Using this dataset, the algorithms change their inner parameters in order to minimize the classification error, through a process called back-propagation. The ResNet architecture achieved the best results on ImageNet Large Scale Visual Recognition Competition (ILSVRC [43]) in 2015, considered the most challenging image 
classification competition. It is still one of the best classification algorithms, while being easy to use and implement.

For the few-shot implementation, we used the Reptile algorithm [39]. Few-shot learning algorithms are specific DL algorithms, whose goal is to be able to fit a model with very few training images. The Reptile algorithm is based on the well-known MAML architecture [33], and more precisely on the first-order version of MAML [48]. The Reptile algorithm is based on the division of the training dataset into a number of tasks $T_{i}$, a task being a learning problem. Through repetitively changing the task during the first training phase (known as meta-training), this algorithm produces a quick learner, i.e. a learner than can quickly adapt to a new task with a small number of examples.

Here, the few-shot algorithms were tested on a classic $n$-ways $k$-shots procedure, $n$ being the number of classes per support set, and $k$ the number of images per class in the support set. For instance, a 5-ways 1shots consists of training 5 classes with supports sets composed of 1 image per classes (e.g. species). We set $n=5$ [34], [36], [40], [42], [49] and allowed $k$ to vary between 1 shot and 30 shots for both experiments 4 and 5 . We did not use data augmentation for FSL experiments for several reasons. First, the goal of FSL is to adapt quickly with a very limited number of images. Second, to have similar settings for method comparison. There were no data-augmentation in the original paper, so we reproduced that. It also allowed us to compare our results with those obtained on benchmarks. Third, the reason behind the use of raw data instead of augmented data in few-shot learning paper is that with very few training samples and few conditions, the risk of overfitting by using the same image modified multiple times is far greater than in classic approaches with important datasets with many conditions. 
253 Model comparison

254 All the DL and FSL models were tested on the independent T2 dataset.

255 First, we compared the results of experiments 1 and 2 in order to

256 estimate the decrease in performance of a classic ResNet DL architecture

257 when trained on a large dataset ATO (i.e. between 11,340 and 73,450

258 images per species after data augmentation, with an average of 3458

259 natural thumbnails per species) or trained on a more limited dataset AT1

260 (i.e. between 400 and 14,360 images per species after data

261 augmentation, with an average of 315 natural thumbnails per species).

262 Second, we compared the results of experiments 1 and 4 in order to

263 evaluate if the ResNet architecture outperforms the Reptile architecture in

264 a real-case situation where thumbnail dataset is large ( TO and ATO).

265 Finally, we compared the results of experiments 2 and 5 to determine

266 whether and to which extent a Reptile model performs better than a

267 ResNet model in a real-case situation where thumbnail dataset is limited ( $T 1$ and $A T 1$ ).

271 In order to better evaluate the performance of ResNet and Reptile algorithms, we also modelled the relationship between model accuracy and the number of thumbnails used to train the models. To achieve this, we fitted the following asymptotic function to the results of experiments 1, 3 and 4 (obtained through training DL and FSL architectures on datasets of various size obtained from ATO and TO): 
where Accuracy $_{\infty}$ is the asymptotic model accuracy when the number of thumbnails $N_{\text {image }}$ is infinite, and $\mathrm{R}$ is the rate at which the asymptote is reached.

Equation 1 was fitted by non-linear mixed-effect modelling (NLME [50]) using species as a random effect. This method is widely used for fitting asymptotic processes. It allows estimating and comparing asymptotic accuracies of both FSL and DL algorithms, and the number of image to reach these asymptotic accuracies. The number of images required to reach the asymptotic accuracy was calculated as the number of images corresponding to an accuracy of 0.99 times the asymptotic value, meaning the asymptote was reached within $1 \%$.

Results

The deep ResNet model trained on the large ATO dataset (3458 natural thumbnails in average per species) during the first experiment obtained a mean accuracy (i.e. percentage of correct classification) of $78.00 \%$ (standard deviation (SD) of $15.16 \%$ ) on $T 2$ test-dataset (Table 2). With this model, accuracy varied among species between $54.14 \%$ (Naso brevirostris) and $99.07 \%$ (Abudefduf vagiensis). The same ResNet DL model trained on smaller AT1 (315 natural thumbnails in average per species) during the second experiment showed highly degraded performance with a mean accuracy of only $42.21 \%(S D=24.95 \%)$. Among species variation ranged with this model from only $3.49 \%$ (Chaetodon trifascialis) to $85.86 \%$ (Chaetodon auriga).

The few-shot Reptile architecture trained on limited T1 dataset during our fifth experiment obtained a mean accuracy of $32.04 \%$ for the 1 -shot learning $(S D=12.70 \%)$ and $51.77 \%$ mean accuracy for the 30 -shots learning $(S D=18.96 \%)$ (Table 2 ,). In this scenario of limited $T 1$ training dataset, the few-shot Reptile algorithm nearly equalled the ResNet DL model with only 5 shots $(41.47 \%$ accuracy for 5 -shots learning on T1 vs $42.21 \%$ for DL on AT1), and performed better beyond 10 shots (45.92\% 
311 of accuracy on T2 with 10-shots learning). A pairwise proportion test

312 showed a $p$-value $<0.0001$, assessing that FSL was significantly better

313 than DL in this scenario beyond 10 shots (Supp. Table 3) accuracy of

314 Reptile models had a standard deviation from $12.70 \%$ with one-shot

315 learning, to $18.96 \%$ with 30 -shots learning, indicating important variation

316 in accuracy among species. However, this standard deviation was smaller

317 than that of the ResNet algorithm trained on the same AT1 limited

318 dataset (24.95\%). 
Figure 1: Relationship between the number of natural thumbnails per species for training and the accuracy of deep-learning and few-shot learning models. Nonlinear mixed effects asymptotic model fit for (a) DL architecture at the fixedeffect level, and for FSL architecture at (b) the fixed-effect level and (c) the random-effect level. Grey areas represent 95\% CI in fixed-effects estimates. Dotted lines represent the NLME estimate of the number of images per species required to reach the $99 \%$ asymptote value. We obtained similar magnitude with the $95 \%$ asymptote value, reached with 750 images for DL and 5 with FSL.

a)

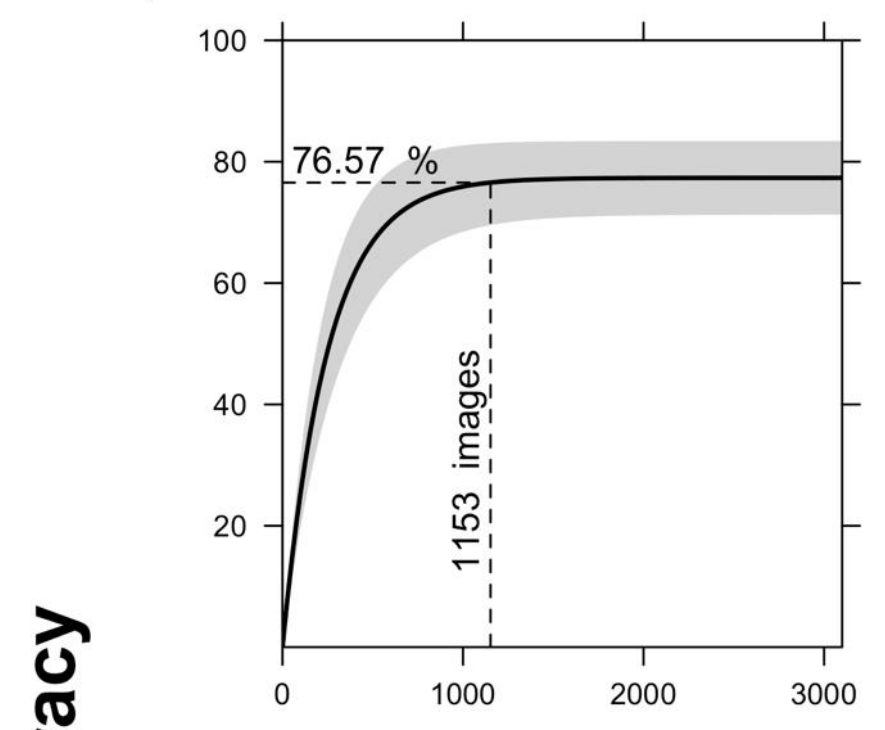

b)

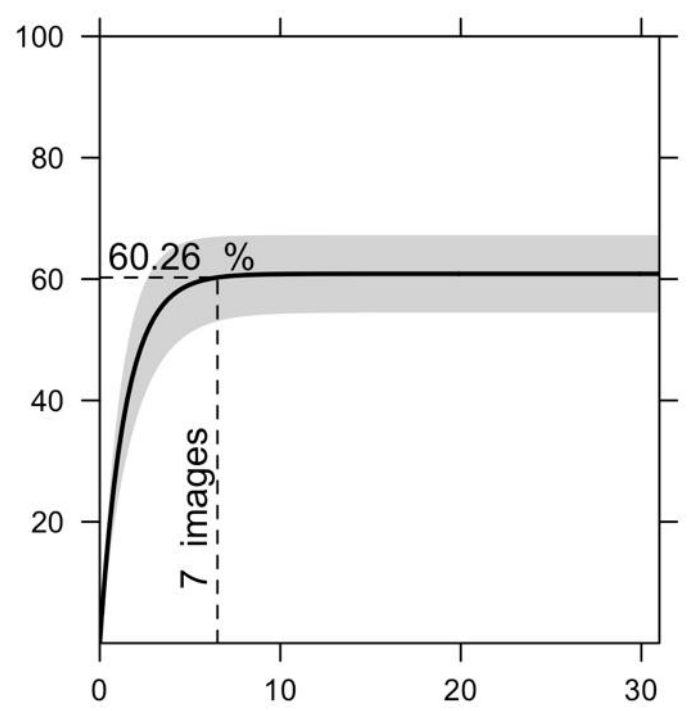

c)

$0 \quad 51015202530$

$0 \quad 51015202530$

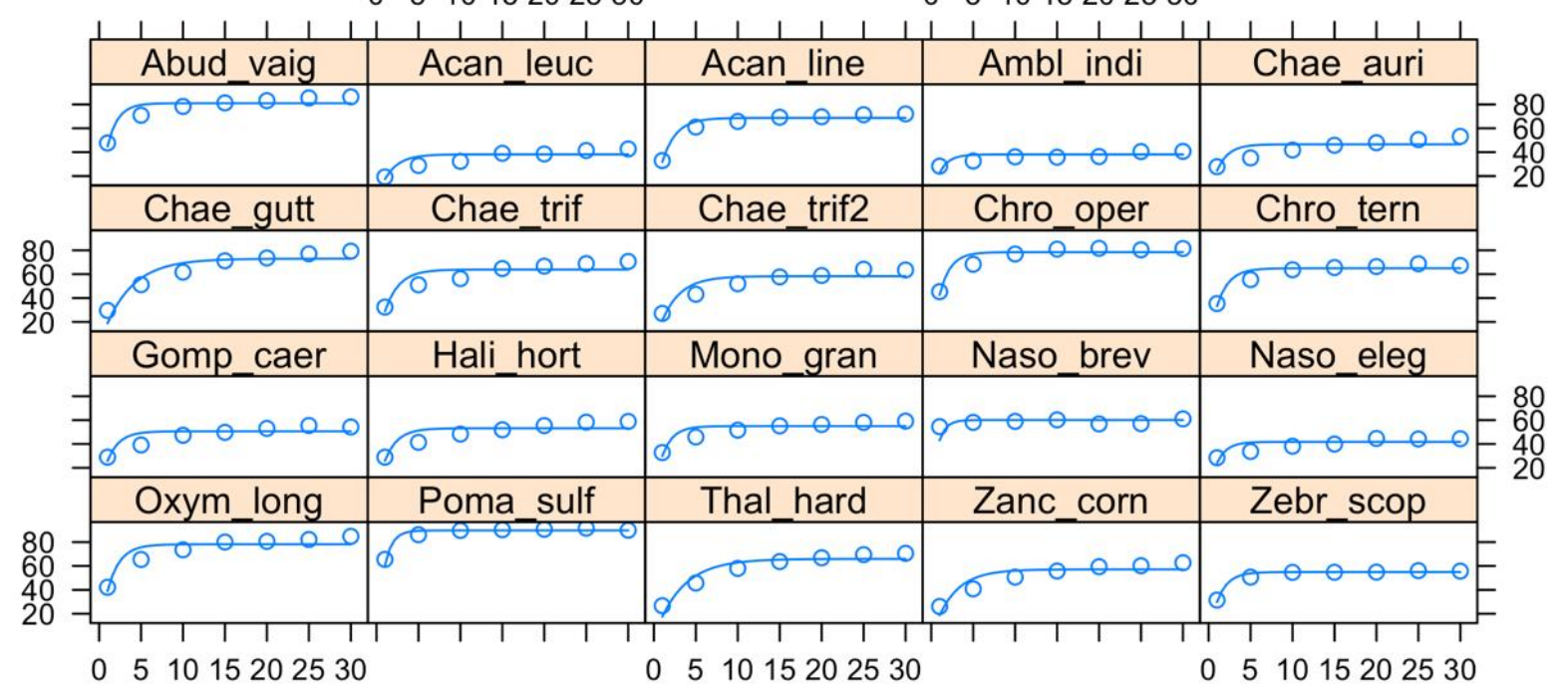


The same few-shot Reptile architecture trained on subsets of T0 during the fourth experiment obtained even better results than when trained on T1, with a mean accuracy on T2 of $34.57 \%$ for 1 -shot, $50.23 \%$ for 5 shots, and up to $64.92 \%$ for 30 -shots (Table 2 ).

Mixed-effects modelling (NLME) of TO and ATO experimental data showed a clear pattern of asymptotic increase of accuracy with the number of natural thumbnails for both Resnet and Reptile architectures (Figure 1).

NLME models included significant species random effect for both DL and FSL (Log-likelihood tests, $\mathrm{P}<0.0001$ ).

The fixed-effect asymptotic value of accuracy was higher for ResNet model (Accuracy $_{\infty}=77.34 \%, 95 \%$ CI: 71.26-83.41\%) than for Reptile model $\left(\right.$ Accuracy $_{\infty}=60.87 \%, 95 \%$ CI: 54.48-67.26\%), illustrating higher classification power of ResNet over Reptile when large numbers of thumbnails are available. However, the slope of the asymptotic model was two-orders of magnitude higher for Reptile $(0.707,95 \%$ CI: 0.5590.854) than for ResNet architecture (0.0040, 95\% CI: 0.0032-0.0048), illustrating the high capacity of Reptile FSL algorithm to learn from only a few images. NLME modelling further showed that average asymptotic accuracy was reached with only 7 natural thumbnails per species for Reptile architecture, compared to 1153 natural thumbnails per species for ResNet, confirming the strong power of Reptile method in situation of limited thumbnail training dataset. However, model random effects showed that some variation existed among species. For Reptile architecture, asymptotic accuracy values ranged from $38.09 \%$ (Amblyglyphidodon indicus) to $89.78 \%$ (Pomacentrus sulfureus), and was reached with 4 to 16 training images per species. For DL architecture, species asymptotes varied from $62.72 \%$ (Monotaxis grandoculis) to 
$35896.81 \%$ (Abudefduf vaigiensis), and could be reached with 786-1776 359 thumbnails per species (Supp. Table 4). 
Table 2: Accuracy of our ResNet deep-learning (DL) and Reptile few-shots learning (FSL) models trained on TO or $T 1$ thumbnails datasets for different number of shots. Accuracy is the \% correct classification of models on T2 test dataset. DL models were trained from TO and T1 after data augmentation (ATO and AT1).

364

\begin{tabular}{|c|c|c|c|c|c|c|c|c|}
\hline \multirow[b]{3}{*}{$\begin{array}{l}\text { Image per species (on } \\
\text { average) }\end{array}$} & \multicolumn{2}{|c|}{$\overline{D L}$} & \multicolumn{6}{|c|}{$\overline{F S L}$} \\
\hline & \multirow{2}{*}{$\begin{array}{c}\text { T0 } \\
3458\end{array}$} & \multirow{2}{*}{$\begin{array}{c}\text { T1 } \\
315\end{array}$} & \multicolumn{3}{|c|}{$\mathrm{T} 1$} & \multicolumn{3}{|c|}{ T0 } \\
\hline & & & 1 shot & 5 shots & 30 shots & 1 shot & 5 shots & 30 shots \\
\hline Abudefduf vaigiensis & 99.07 & 69.91 & 16.08 & 11.39 & 11.38 & 47.67 & 70.9 & 86.35 \\
\hline Acanthurus leucosternon & 86.15 & 44.67 & 25.51 & 30.71 & 38.74 & 19.23 & 28.8 & 42.66 \\
\hline Acanthurus lineatus & 59.72 & 20.37 & 39.86 & 56.04 & 72.50 & 32.93 & 61.01 & 72.02 \\
\hline Amblyglyphidodon indicus & 58.78 & 60.78 & 25.75 & 26.74 & 32.86 & 28.26 & 32.55 & 40.64 \\
\hline Chaetodon auriga & 87.05 & 85.86 & 18.16 & 25.68 & 36.56 & 27.8 & 35.18 & 53.20 \\
\hline Chaetodon guttatissimus & 85.50 & 44.12 & 33.58 & 44.21 & 58.26 & 29.61 & 51.18 & 79.29 \\
\hline Chaetodon trifascialis & 90.00 & 3.49 & 29.02 & 25.48 & 28.44 & 27.14 & 43.17 & 63.51 \\
\hline Chaetodon trifasciatus & 87.80 & 28.05 & 38.73 & 50.63 & 66.72 & 32.41 & 51.07 & 70.63 \\
\hline
\end{tabular}




\begin{tabular}{|c|c|c|c|c|c|c|c|c|}
\hline Chromis opercularis & 61.29 & 9.68 & 44.01 & 61.81 & 62.94 & 45.34 & 68.28 & 81.50 \\
\hline Chromis ternatensis & 59.61 & 55.77 & 18.91 & 24.94 & 35.07 & 35.4 & 55.44 & 67.22 \\
\hline Gomphosus caeruleus & 75.72 & 20.81 & 26.01 & 38.99 & 58.74 & 28.96 & 39.16 & 54.22 \\
\hline Halichoeres hortulanus & 82.93 & 17.07 & 31.82 & 44.81 & 57.01 & 28.94 & 41.35 & 58.87 \\
\hline Monotaxis grandoculis & 57.10 & 53.37 & 32.03 & 41.52 & 50.64 & 32.8 & 45.85 & 59.13 \\
\hline Naso brevirostris & 54.14 & 68.60 & 47.06 & 54.26 & 64.66 & 54.47 & 58.08 & 61.00 \\
\hline Naso elegans & 93.24 & 79.43 & 34.54 & 43.11 & 52.17 & 28.47 & 33.71 & 44.36 \\
\hline Oxymonacanthus & 96.43 & 14.54 & 39.29 & 53.48 & & 42.15 & 65.44 & \\
\hline longirostris & & & & & 66.26 & & & 84.86 \\
\hline Pomacentrus sulfureus & 90.14 & 61.97 & 70.90 & 88.18 & 93.93 & 65.53 & 86.21 & 90.00 \\
\hline Thalassoma hardwicke & 90.90 & 51.64 & 25.64 & 44.4 & 67.96 & 26.72 & 45.7 & 70.60 \\
\hline Zanclus cornutus & 81.36 & 40.68 & 18.33 & 31.28 & 44.44 & 26.08 & 40.82 & 62.72 \\
\hline Zebrasoma scopas & 63.04 & 13.30 & 25.56 & 31.81 & 36.05 & 31.42 & 50.69 & 55.70 \\
\hline MEAN & 78.00 & 42.21 & 32.04 & 41.47 & 51.77 & 34.57 & 50.23 & 64.92 \\
\hline SD & 15.16 & 24.95 & 12.70 & 16.93 & 18.96 & 11.14 & 14.75 & 14.55 \\
\hline
\end{tabular}




\section{Discussion}

Our experiments demonstrated that few-shot learning methods based on Reptile architecture can be effectively used to drastically reduce the number of annotated images for underwater fish identification. Accuracy levels obtained with few-shot learning algorithm trained with only five training images are close to those of a standard Deep Learning architecture such as ResNet trained with 400-14350 images per species. Further, FSL architecture trained with 10 images outperformed a ResNet 100 architecture trained with at least 400 images per species. This is a very promising result in situations where many species need to be identified from models trained with a few images, a typical characteristic in marine biodiversity applications.

However, the important standard deviation among the different trained species (18.96 SD on 30-shots) showed that few-shot algorithms may not be robust enough to discriminate among similar species showing only subtle differences. Nevertheless, in our $2^{\text {nd }}$ experiment, our ResNet model achieved an accuracy under $40 \%$ for all the species with fewer training images than 1140 (after data augmentation, i.e. 114 natural images), and only 7 species were identified with an accuracy greater than $45 \%$. These species were represented with a range of 2700-14350 images during the training phase. We also show better results with the model trained on T0 than the model trained on T1. As expected, increasing the number of images per shot rely on better performances as well as increasing the per species images variability. However, in real conditions, few-shot learning is to be used in a context where very few images per classes are at disposal. Therefore, the dataset T1 corresponded more to a real use case scenario.

Thus, there is a trade-off to make between accuracy and robustness on one hand, and the cost of video annotation by experts on the other. 
Modeling the accuracy of neural networks using NLME allowed to understand the number of images per species required for the Few-shot and Deep architecture to reach $99 \%$ of their maximum potential accuracy. In our case study, there was a 150-fold factor between the average number of images required for a Deep Learning architecture (1153 images) and for a Few-shot architecture (7) to reach asymptotic accuracy. However, it is important to note that these numbers could vary according to the number and complexity of classes fed to the deep classifier.

In this work we used a Reptile FSL architecture. As the field of few-shot learning is quickly improving, new methods are proposed at a fast rate. While Reptile obtained a mean accuracy of $61.98 \%$ on the MiniImageNet dataset (the most used benchmark for few-shot learning methods) through a 5-shots learning, [51] recently achieved $80.51 \%$ of accuracy on the same dataset. Although further studies are required, we can reasonably assume that the improvements of FSL algorithms will further expand the possible use of few-shot learning for real-life use cases.

Applied to marine and coral reef ecology, such methods requiring few examples to fit a model on an identification task could be used for studies on species rarely seen on screen. A key characteristic of highly diverse ecosystems is that they are composed of few very common species and a large proportion of less-common and rare species. Hence, the important effort required to build databases with a sufficient number of images of all these rare species is the main bottleneck preventing the use of Deep Learning on a large number of species. The improvement of few-shot learning algorithms offers promises to build efficient identification models to automatically process images and videos to localise and identify rare fish species. Such models could then be paired with more classic deep architectures, more efficient to identify abundant species with the leverage of important datasets.

Acknowledgements: 
423 This study was funded by the French National Research Agency project ANR 424 18-CE02-0016 SEAMOUNTS.

425

426

427

428 
430 References

431 [1] R. Dirzo, H. S. Young, M. Galetti, G. Ceballos, N. J. B. Isaac, and B. Collen, 432 "Defaunation in the Anthropocene," Science (80-. )., vol. 345, no. 6195, pp. $433 \quad 401-406,2014$.

[2] H. S. Young, D. J. Mccauley, M. Galetti, and R. Dirzo, "Patterns, Causes, and Consequences of Anthropocene Defaunation," Annu. Rev. Ecol. Evol. Syst., no. August, pp. 333-358, 2016.

[3] J. E. Cinner et al., "Meeting fisheries, ecosystem function, and biodiversity goals in a human-dominated world," Science (80-. )., vol. 311, no. April, pp. 307-311, 2020.

[4] R. D. Stuart-smith et al., "Integrating abundance and functional traits reveals new global hotspots of fish diversity," Nature, vol. 501, no. 7468, pp. 539$542,2013$.

[5] A. Heenan et al., "Long-term monitoring of coral reef fish assemblages in the Western central pacific," Sci. Data, vol. 4, pp. 1-12, 2017.

[6] S. K. Whitmarsh, P. G. Fairweather, and C. Huveneers, "What is Big BRUVver up to ? Methods and uses of baited underwater video," Rev. Fish Biol. Fish., vol. 27, no. 1, pp. 53-73, 2017.

[7] M. Aaron MacNeil et al., "Global status and conservation potential of reef sharks," Nature, no. July 2019, 2020.

[8] J. Juhel, L. Vigliola, L. Wantiez, T. B. Letessie, J. J. Meeuwig, and D. Mouillot, "Isolation and no-entry marine reserves mitigate anthropogenic impacts on grey reef shark behavior," Sci. Rep., vol. 9, no. November 2018, pp. 1-11, 2019.

[9] M. Cappo, G. De, and P. Speare, "Inter-reef vertebrate communities of the Great Barrier Reef Marine Park determined by baited remote underwater video stations," Mar. Ecol. Prog. Ser., vol. 350, pp. 209-221, 2007. 
[10] V. Zintzen, M. J. Anderson, C. D. Roberts, E. S. Harvey, and L. Andrew, "Effects of latitude and depth on the beta diversity of New Zealand fish communities," Sci. Rep., vol. 7, no. July, pp. 1-10, 2017.

[11] Tom B Letessier et al., "Remote reefs and seamounts are the last refuges for marine predators across the Indo- Pacific," PLOS Biol., vol. 17, pp. 1-20, 2019.

[12] C. J. Torney et al., "A comparison of deep learning and citizen science techniques for counting wildlife in aerial survey images," Methods Ecol. Evol., vol. 10, no. October 2018, pp. 779-787, 2019.

[13] E. C. Mcclure et al., "Artificial Intelligence Meets Citizen Science to Supercharge Ecological Monitoring," Patterns, vol. 1, no. 7, p. 100109, 2020.

[14] M. Willi et al., "Identifying animal species in camera trap images using deep learning and citizen science," Methods Ecol. Evol., vol. 10, no. 1, pp. 80-91, 2019.

[15] Z. Miao et al., "Insights and approaches using deep learning to classify wildlife," Sci. Rep., no. May, pp. 1-9, 2019.

[16] M. Lasseck, "Audio-based Bird Species Identification with Deep Convolutional Neural Networks Audio-based Bird Species Identification with Deep Convolutional Neural Networks," no. January, 2020.

[17] Y. Shiu et al., "Deep neural networks for automated detection of marine mammal species," pp. 1-12, 2020.

[18] D. Rathi, S. Jain, and S. Indu, "Underwater Fish Species Classification using Convolutional Neural Network and Deep Learning. (arXiv:1805.10106v1 [cs.CV])," no. June, 2018.

[19] A. Salman et al., "OCEANOGRAPHY : METHODS Fish species classification in unconstrained underwater environments based on deep learning," pp. 570585, 2016.

[20] S. Villon et al., "A Deep Learning algorithm for accurate and fast identification 
of coral reef fishes in underwater videos," PeerJ Prepr., vol. 6, p. e26818v1, 2018.

[21] H. Qin, X. Li, J. Liang, Y. Peng, and C. Zhang, "DeepFish: Accurate underwater live fish recognition with a deep architecture," Neurocomputing, vol. 187, pp. 49-58, 2016.

[22] P. Chabanet, S. R. Floeter, A. Friedlander, J. Mcpherson, and R. E. Myers, "Global Biogeography of Reef Fishes: A Hierarchical Quantitative Delineation of Regions," PLoS One, vol. 8, no. 12, 2013.

[23] A. P. Hercos, M. Sobansky, H. L. Queiroz, A. E. Magurran, and A. Andre, "Local and regional rarity in a diverse tropical fish assemblage," Biol. Sci., vol. 280, pp. 81-101, 2013.

[24] G. E. Jones, M. J. Caley, and P. L. Munday, "Rarity in Coral Reef Fish Communities," Coral reef fishes Dyn. Divers. a complex Ecosyst., pp. 88-101, 2002.

[25] L. Liu, T. Zhou, G. Long, J. Jiang, and C. Zhang, "Many-Class Few-Shot Learning on Multi-Granularity Class Hierarchy," IEEE Trans. Knowl. Data Eng., pp. 1-14, 2020.

[26] A. Li, T. Luo, Z. Lu, T. Xiang, and L. Wang, "Large-Scale Few-Shot Learning: Knowledge Transfer With Class Hierarchy," in Proceedings of the IEEE Conference on Computer Vision and Pattern Recognition, 2019, pp. 72127220.

[27] P. Zhuang, Y. Wang, and Y. Qiao, "WildFish : A Large Benchmark for Fish Recognition in the Wild," in Proceedings of the 26th ACM international conference on Multimedia, 2018, vol. 2, pp. 1301-1309.

[28] J. Wang and L. Perez, "The Effectiveness of Data Augmentation in Image Classification using Deep Learning," arXiv Prepr. arXiv1712.04621., 2017.

[29] D. A. Van Dyk and X. Meng, "The Art of Data Augmentation," J. Comput. Graph. Stat., vol. 8600, no. 2001, pp. 1-50, 2012. 
[30] S. C. Wong, M. D. Mcdonnell, G. Adam, and S. Victor, "Understanding data augmentation for classification: when to warp?," in 2016 international conference on digital image computing: techniques and applications (DICTA), 2016, pp. 1-6.

[31] L. Fei-fei, R. Fergus, S. Member, and P. Perona, "One-Shot Learning of Object Categories," EEE Trans. pattern Anal. Mach. Intell., vol. 28, no. 4, pp. 594$611,2006$.

[32] M. Fink, "Object Classification from a Single Example Utilizing Class Relevance Metrics," in Advances in neural information processing systems, 2005, pp. 449-456.

[33] C. Finn, P. Abbeel, and S. Levine, "Model-Agnostic Meta-Learning for Fast Adaptation of Deep Networks," arXiv Prepr. arXiv1703.03400, 2017.

[34] F. Sung, Y. Yang, and L. Zhang, "Learning to Compare: Relation Network for Few-Shot Learning Queen Mary University of London," in Proceedings of the IEEE/CVF Conference on Computer Vision and Pattern Recognition (CVPR), 2018, pp. 1199-1208.

[35] Liu Yanbin et al., "Learning to proagate labels: Transductive propagation network for few-shot learning," in arXiv preprint arXiv:1805.10002, 2019, pp. $1-14$.

[36] J. Victor, Garcia Bruna, "FEW-SHOT LEARNING WITH GRAPH NEURAL NETWORKS," in arXiv preprint arXiv:1711.04043, 2017., 2018, pp. 1-13.

[37] S. Gidaris, P. Paristech, N. Komodakis, and P. Paristech, "Dynamic Few-Shot Visual Learning without Forgetting," in Proceedings of the IEEE Conference on Computer Vision and Pattern Recognitio, 2018, pp. 4367-4375.

[38] B. Hariharan, R. Girshick, and F. Ai, "Low-shot Visual Recognition by Shrinking and Hallucinating Features," in Proceedings of the IEEE International Conference on Computer Vision, 2017, pp. 3018-3027.

[39] A. Nichol and J. Schulman, "Reptile : a Scalable Metalearning Algorithm," arXiv Prepr. arXiv1803.02999, 2018, pp. 1-11, 2018. 
[40] Q. Sun and Y. L. T. Chua, "Meta-Transfer Learning for Few-Shot Learning," Conf. Comput. Vis. Pattern Recognit., pp. 403-412, 2018.

[41] Y. Wang, Q. Yao, J. T. Kwok, and L. M. Ni, "Generalizing from a Few Examples: A Survey on Few-Shot Learning arXiv : 1904 . 05046v2 [ cs . LG ] 13 May 2019," 2019.

[42] M. A. Jamal and H. Cloud, "Task Agnostic Meta-Learning for Few-Shot Learning," in Proceedings of the IEEE/CVF Conference on Computer Vision and Pattern Recognition (CVPR), 2019.

[43] O. Russakovsky et al., "ImageNet Large Scale Visual Recognition Challenge," Int. J. Comput. Vis., pp. 211-252, 2015.

[44] B. M. Lake, R. Salakhutdinov, and J. B. Tenenbaum, "The Omniglot challenge: a 3-year progress report," COBEHA, vol. 29, pp. 97-104, 2019.

[45] K. He, X. Zhang, S. Ren, and J. Sun, "Deep Residual Learning for Image Recognition," in Proceedings of the IEEE conference on computer vision and pattern recognition, 2016, pp. 770-778.

[46] S. Villon, D. Mouillot, M. Chaumont, and G. Subsol, "A new method to control error rates in automated species identification with deep learning algorithms," Sci. Rep., vol. 10, pp. 1-13, 2020.

[47] Y. Lecun, Y. Bengio, and G. Hinton, "Deep learning," Nature, pp. 436-444, 2015.

[48] A. Nichol, J. Achiam, and J. Schulman, "On First-Order Meta-Learning Algorithms," arXiv, pp. 1-15, 2018.

[49] Y. Wang, Q. Yao, and L. M. Ni, "Generalizing from a Few Examples: A Survey on Few-shot Generalizing from a Few Examples: A Survey on Few-shot," ACM Comput. Surv., vol. 53, no. June, 2020.

[50] J. Pinheiro and D. Bates, Mixed-effects models in S and S-PLUS. 2006.

[51] H. Li, D. Eigen, S. Dodge, M. Zeiler, and X. Wang, "Finding Task-Relevant Features for Few-Shot Learning by Category Traversal," in Proceedings of the 

2019, vol. 1.

572

573

574

575 


\begin{tabular}{|c|c|c|c|}
\hline $\begin{array}{l}\text { Abudefduf } \\
\text { vaigiensis }\end{array}$ & $\begin{array}{l}\text { Acanthurus } \\
\text { leucosternon }\end{array}$ & $\begin{array}{l}\text { Acanthurus } \\
\text { lineatus }\end{array}$ & $\begin{array}{l}\text { Amblyglyphidodon } \\
\text { indicus }\end{array}$ \\
\hline 1 & 6) & & \\
\hline Chaetodon auriga & $\begin{array}{l}\text { Chaetodon } \\
\text { guttatissimus }\end{array}$ & $\begin{array}{l}\text { Chaetodon } \\
\text { trifascialis }\end{array}$ & $\begin{array}{l}\text { Chaetodon } \\
\text { trifasciatus }\end{array}$ \\
\hline & & & \\
\hline $\begin{array}{l}\text { Chromis } \\
\text { opercularis }\end{array}$ & $\begin{array}{l}\text { Chromis } \\
\text { ternatensis }\end{array}$ & $\begin{array}{l}\text { Gomphosus } \\
\text { caeruleus }\end{array}$ & $\begin{array}{l}\text { Halichoeres } \\
\text { hortulanus }\end{array}$ \\
\hline
\end{tabular}




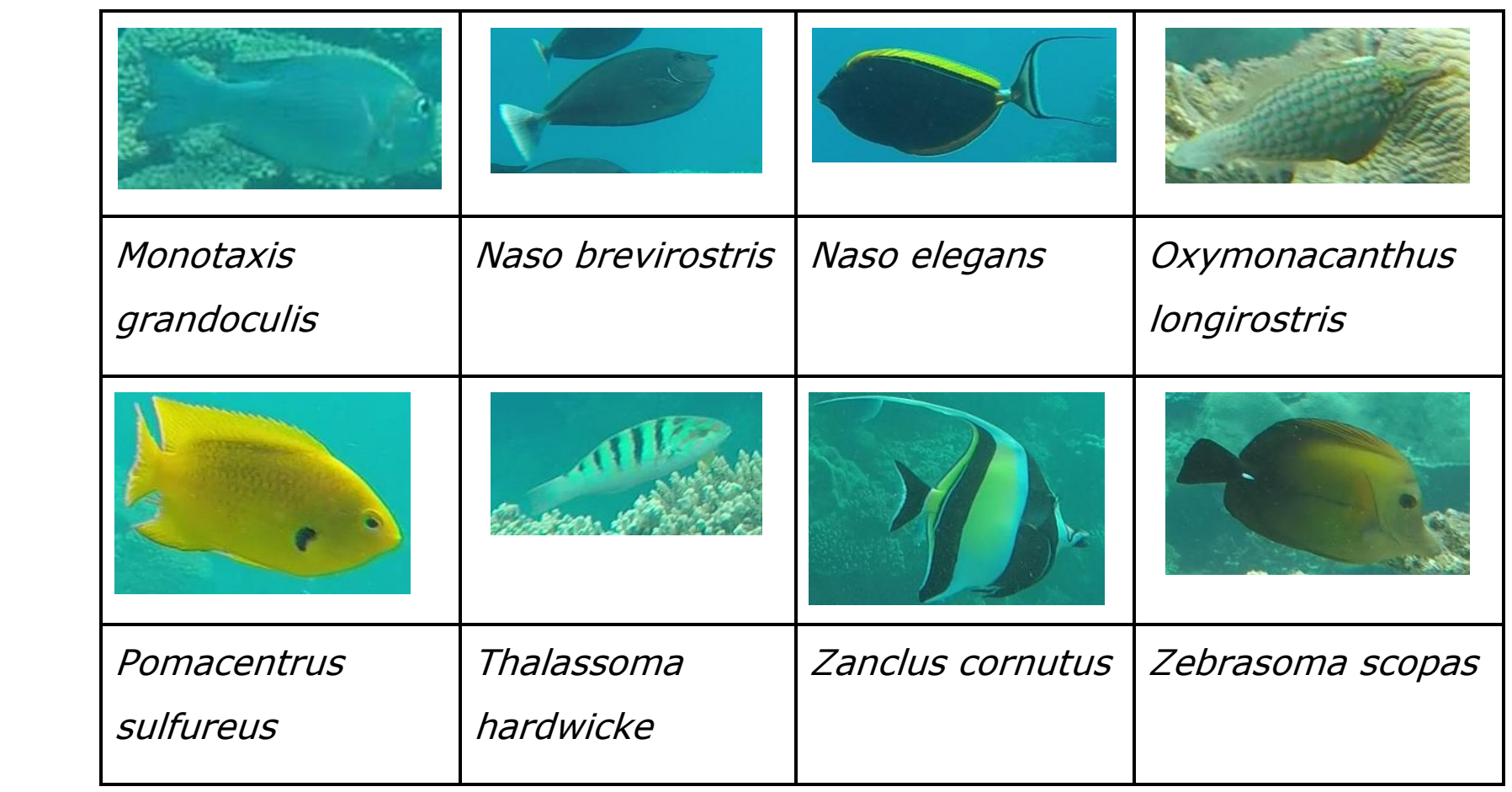

Supp. Fig. 1: The 20 reef fish species considered in this study 

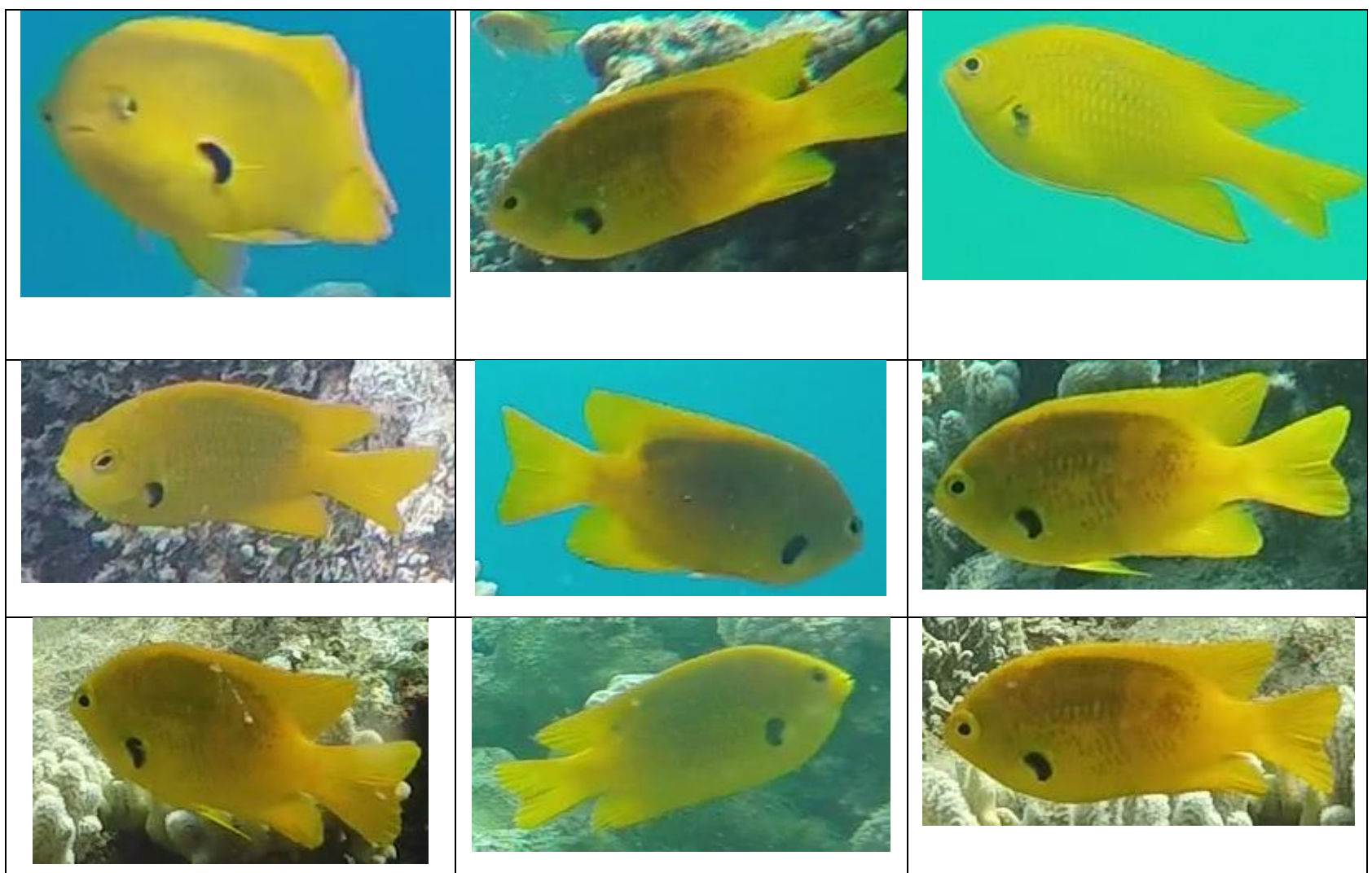

Supp. Fig. 2: Diversity of individuals of the same species and of their
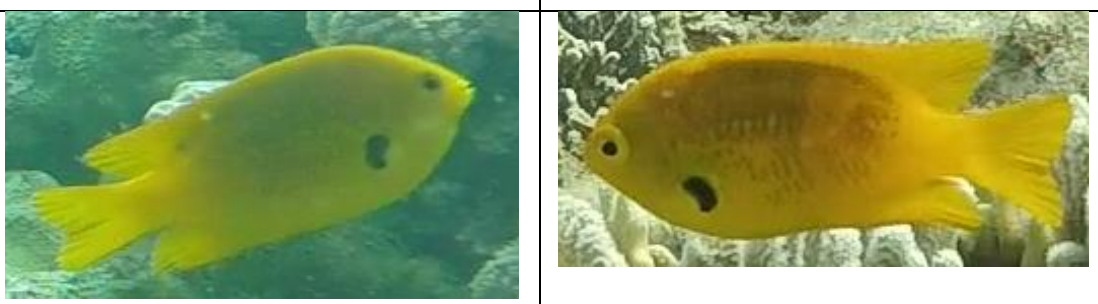

586 environments.

587 


\begin{tabular}{|l|l|l|}
\hline & & \\
\hline & & \\
\hline
\end{tabular}

Supp. Fig. 3: Examples of classes' images in MiniImageNet

Supp. Table 1: Dataset usage during our experiments

\begin{tabular}{llrl}
\hline $\begin{array}{l}\text { Dataset } \\
\text { name }\end{array}$ & Building & Number of annotations & Usage \\
\hline T0 & Human Annotation & 69,169 & $\begin{array}{l}\text { Building of supports sets of 1,5,15 and 30 } \\
\text { images for our fourth experiment } \\
\end{array}$ \\
& $\begin{array}{c}\text { Building of supports sets or 1, 5, 15 and } \\
\text { T1 }\end{array}$ & 6,320 & 30 images for our fifth experiment \\
T2 & Human Annotation & 13,232 & testing dataset \\
AT0 & Human Annotation & 691,690 & DL training for our first experiment \\
AT1 & Data augmentation applied on T0 & 63,200 & DL training for our second experiment \\
\hline
\end{tabular}

593 Supp. Table 2: Mean accuracy obtained with FSL models trained with $5941,5,10,15,20,25$ and 30 images per species. All the images used for the 595 supports set are from $\mathrm{T} 1$.

\section{Number of thumbnails in the Support set}

\begin{tabular}{lrrrrrrr}
\hline Species & 1 & 5 & 10 & 15 & 20 & 25 & 30 \\
\hline Abudefduf vaigiensis & 16.08 & 11.39 & 13.18 & 12.59 & 14.02 & 12.98 & 11.38 \\
Acanthurus & & & & & & & \\
leucosternon & 25.51 & 30.71 & 34.90 & 36.68 & 37.16 & 36.95 & 38.74 \\
Acanthurus lineatus & 39.86 & 56.04 & 63.52 & 66.21 & 70.02 & 70.93 & 72.50
\end{tabular}


Amblyglyphidodon

indicus

$\begin{array}{lllllll}25.75 & 26.74 & 28.06 & 28.80 & 30.24 & 32.20 & 32.86\end{array}$

$\begin{array}{llllllll}\text { Chaetodo auriga } & 18.16 & 25.68 & 30.67 & 32.63 & 33.78 & 35.42 & 36.56\end{array}$

Chaetodon

guttatissimus

$\begin{array}{lllllll}33.58 & 44.21 & 47.40 & 49.26 & 54.12 & 55.91 & 58.26\end{array}$

Chaetodon trifascialis

29.02

$25.48 \quad 27.4$

28.02

$28.18 \quad 29.65 \quad 28.44$

Chaetodon

trifasciatus

$\begin{array}{lllllll}38.73 & 50.63 & 53.47 & 60.17 & 63.19 & 64.38 & 66.72\end{array}$

Chromis opercularis

44.01

$61.81 \quad 61.85$

63.85

64.67

$61.98 \quad 62.94$

Chromis ternatensis

18.9

24.94

26.27

31.2

$33.87 \quad 33.49 \quad 35.07$

Gomphosus

caeruleus

$\begin{array}{lllllll}26.01 & 38.99 & 46.84 & 52.88 & 53.96 & 58.69 & 58.74\end{array}$

Halichoeres

hortulanus

$\begin{array}{lllllll}31.82 & 44.81 & 50.73 & 53.52 & 54.11 & 55.85 & 57.01\end{array}$

Monotaxis

grandoculis

$\begin{array}{lllllll}32.03 & 41.52 & 45.68 & 47.59 & 48.34 & 49.19 & 50.64\end{array}$

Naso brevirostris

47.06

$54.26 \quad 61.01$

59.46

59.30

$62.40 \quad 64.66$

Naso elegans

34.54

43.11

48.38

50.1

$51.31 \quad 50.55 \quad 52.17$

Oxymonacanthus

longirostris

$\begin{array}{lllllll}39.29 & 53.48 & 59.71 & 58.84 & 62.70 & 64.37 & 66.26\end{array}$

Pomacentrus

sulfureus

70.90

88.1890 .92

93.08

92.67

94.1393 .93

Thalassoma

hardwicke

$\begin{array}{lllllll}25.64 & 44.40 & 57.80 & 62.03 & 64.97 & 67.86 & 67.96\end{array}$

Zanclus cornutus

$\begin{array}{lllllll}18.33 & 31.28 & 37.27 & 41.71 & 41.70 & 42.95 & 44.44\end{array}$ 


\begin{tabular}{llllllll} 
Zebrasoma scopas & 25.56 & 31.81 & 33.32 & 34.62 & 37.02 & 37.22 & 36.05 \\
\hline Mean & & & & & & & \\
SD & 32.04 & 41.47 & 45.92 & 48.17 & 49.77 & 50.86 & 51.77 \\
& 12.70 & 16.93 & 17.69 & 18.00 & 18.10 & 18.52 & 18.96
\end{tabular}

596

597 
598 Supp. Table 3: Probability values of the pairwise proportional test used to

599 assess the significance of the difference between accuracies obtained

600 through few-shot models with 5, 10, 15, 20, 25 and 30 shots and deep

601 learning model trained on T1.

\begin{tabular}{cccccccc}
\hline \multicolumn{1}{c}{ DL } & FS1 & FS5 & FS10 & FS15 & FS20 & FS25 \\
\hline FS1 & $<2 \mathrm{e}-16$ & & & & & & \\
FS5 & 0.28 & $<2 \mathrm{e}-16$ & & & & & \\
FS10 & $1.20 \mathrm{E}-08$ & $<2 \mathrm{e}-16$ & $3.80 \mathrm{E}-12$ & & & & \\
FS15 & $<2 \mathrm{e}-16$ & $<2 \mathrm{e}-16$ & $<2 \mathrm{e}-16$ & 0.0016 & & & \\
FS20 & $<2 \mathrm{e}-16$ & $<2 \mathrm{e}-16$ & $<2 \mathrm{e}-16$ & $4.10 \mathrm{E}-09$ & 0.0379 & & \\
FS25 & $<2 \mathrm{e}-16$ & $<2 \mathrm{e}-16$ & $<2 \mathrm{e}-16$ & $1.30 \mathrm{E}-14$ & $8.90 \mathrm{E}-05$ & 0.2362 & \\
FS30 & $<2 \mathrm{e}-16$ & $<2 \mathrm{e}-16$ & $<2 \mathrm{e}-16$ & $<2 \mathrm{e}-16$ & $3.90 \mathrm{E}-08$ & 0.0059 & 0.28 \\
\hline
\end{tabular}

602

603 Supp. Table 4: Value of the asymptotic accuracy predicted by the NLME

604 models, and number of natural images required for both Deep Learning

605 architecture and Few-shot Learning architecture to reach 99\% of this

606 asymptote.

Deep Learning

Number of images

required to reach

$99 \%$ of the

asymptote.
Few-Shot Learning

Number of images

Accuracy required to reach

asymptote $\quad 99 \%$ of the

Accuracy

asymptote. asymptote value

\begin{tabular}{|c|c|c|c|c|}
\hline Naso brevirostris & $1,506.47$ & 67.43 & 5.04 & 38.10 \\
\hline \multicolumn{5}{|l|}{ Monotaxis } \\
\hline grandoculis & $1,769.71$ & 62.72 & 7.59 & 38.14 \\
\hline \multicolumn{5}{|l|}{ Amblyglyphidodon } \\
\hline indicus & $1,766.55$ & 62.89 & 5.34 & 41.76 \\
\hline \multicolumn{5}{|l|}{ Chromis } \\
\hline ternatensis & $1,492.65$ & 67.75 & 6.16 & 46.55 \\
\hline Acanthurus & $1,425.04$ & 69.30 & 6.42 & 50.59 \\
\hline
\end{tabular}


lineatus

Zebrasoma scopas

$1,776.17$

62.74

5.92

54.94

Gomphosus

caeruleus

$1,341.80$

71.51

6.90

53.15

Chromis

opercularis

$1,112.28$

78.67

5.85

54.95

Zanclus cornutus

$1,069.22$

80.57

3.66

60.08

Halichoeres

hortulanus

$1,250.29$

74.18

11.60

57.14

Acanthurus

leucosternon

$1,184.97$

76.31

10.21

58.28

Chaetodon

guttatissimus

868.64

90.93

6.46

64.95

Chaetodon auriga

$1,053.06$

81.31

14.97

65.94

Chaetodon

trifasciatus

$1,076.99$

80.34

7.70

63.88

Chaetodon

trifascialis

$1,087.93$

79.94

7.42

68.66

Pomacentrus

sulfureus

960.99

85.63

15.41

72.98

Thalassoma

hardwicke

994.27

84.00

5.86

78.42

Naso elegans

$1,074.08$

80.48

6.66

78.13

Oxymonacanthus

longirostris

833.75

93.29

5.68

80.97

Abudefduf

vaigiensis

785.95

96.82

4.12

89.78

Mean

1221.54

77.34

7.45

60.87

607

608 
609 